\title{
Associations of chemo- and radio-resistant phenotypes with the gap junction, adhesion and extracellular matrix in a three-dimensional culture model of soft sarcoma
}

Chujie Bail ${ }^{1+}$ Min Yang ${ }^{2+}$, Zhengfu Fan ${ }^{1}$, Shu Li ${ }^{1}$, Tian Gao ${ }^{1}$ and Zhiwei Fang ${ }^{1 *}$

\begin{abstract}
Background: Three-dimensional (3D) culture models are considered to recapitulate the cell microenvironment in solid tumors, including the extracellular matrix (ECM), cell-cell interactions, and signal transduction. These functions are highly correlated with cellular behaviors and contribute to resistances against chemo- and radio-therapies. However, the biochemical effects and mechanisms remain unknown in soft sarcoma. Therefore, we developed an in vitro 3D model of sarcoma to analyze the reasons of the chemo- and radio-resistance in therapies.

Methods: Four soft sarcoma cell lines, HT1080, RD, SW872, and human osteosarcoma cell line 1 (HOSS1), a cell line established from a patient-derived xenograft, were applied to 3D culture and treated with growth factors in methylcellulose-containing medium. Spheroids were examined morphologically and by western blotting, RT-qPCR, and immunofluorescence staining to analyze cell adhesion, gap junctions, ECM genes, and related factors. Proliferation and colony formation assays were performed to assess chemo- and radio-resistances between 3D and two-dimensional (2D) cell cultures. Annexin V and Propidium lodide staining was used to detect early apoptotic sarcoma cells treated with Doxorubicin, Gemcitabine, and Docetaxel in the 3D model.
\end{abstract}

Results: The four soft sarcoma cell lines formed spheres in vitro by culture in modified condition medium. Compared with 2D cell culture, expression of ECM genes and proteins, including COLIA1, LOX, SED1, FN1, and LAMA4, was significantly increased in 3D culture. Analysis of cadherin and gap junction molecules showed significant changes in the gene and protein expression profiles under 3D conditions. These changes affected cell-cell communication and were mainly associated with biological processes such as cell proliferation and apoptosis related to chemo- and radio-resistances.

Conclusions: Our findings revealed significant differences between 3D and 2D cell culture systems, and indicated that cellular responsiveness to external stress such as radiation and chemotherapeutics is influenced by differential expression of genes and proteins involved in regulation of the ECM, cell adhesion, and gap junction signaling.

Keywords: Soft sarcoma, Three-dimensional culture, Primary culture, Human osteosarcoma cell line 1 (HOSS1), Chemo-resistance, Radio-resistance

\footnotetext{
*Correspondence: zhiwei_fang66@163.com

${ }^{\dagger}$ Equal contributors

'Department Bone and Soft Tissue Tumor, Key Laboratory of Carcinogenesis and Translational Research (Ministry of Education), Peking University Cancer Hospital and Institute, Beijing 100142, People's Republic of China Full list of author information is available at the end of the article
} 


\section{Introduction}

Soft tissue sarcomas are malignant tumors of mesenchymal origin, among which rhabdomyosarcoma [1], fibrosarcoma [2], and osteosarcoma [3], are the most common histological subtypes in childhood and adolescence. Despite advances in cancer therapy, the mainstay of treatment for this disease is still surgical resection. Despite conventional chemotherapy and radiation therapy, the overall survival of patients with soft sarcomas has not improved in the past 50 years $[4,5]$. The prognosis of soft sarcomas remains one of the worst amongst solid tumors in children [6]; consequently, soft sarcoma has become an increasingly researched cancer over the past few decades.

Traditionally, cancer researchers rely on twodimensional (2D) in vitro studies and small animal models to examine the complex mechanisms of cancer cell proliferation, apoptosis, angiogenesis, invasion, and metastasis. Typically, the arrangement of the cells in 2D primary culture permits less cell-cell interaction and absent ECM secretion, with concomitant effects on gene and protein expression and cellular behavior [7, 8]. Therefore, a novel method of culturing tumor cells, three-dimensional (3D) culture, was introduced in the 1970s [9]. This model allowed researches to focus on the cellular morphology and interactions between tumor cells. Cell-cell and cellECM interactions observed during in vivo tumor progression cannot be studied in 2D models, whereas 3D models are capable of mimicking these conditions. Subsequently, various sarcoma cell lines have been tested for their ability to grow as spheroids in 3D culture [10-12]. Many studies have verified that 3D cultures of epithelial tumor cell lines are generally more resistant to chemo- and radio-therapies than their 2D counterparts [13-15]. Chemo-resistance in tumors is mediated by various mechanisms. The classical mechanisms are based on ATP-binding cassette $(\mathrm{ABC})$ transporter proteins, particularly $\mathrm{ABCB} 1$ (MDR1), ABCC1 (MRP1), and ABCG2 (BCRP1), all of which have been reported to contribute to chemoresistance in soft sarcoma cells [16]. However, validated 3D in vitro sarcoma cell models have not been developed for rapid and standardized screening of chemo- and radio-resistances.

In the present study, we developed an in vitro alginate scaffold 3D model of sarcoma using HT1080 (fibrosarcoma), SW872 (liposarcoma), and RD (rhabdomyosarcoma) cell lines, as well as human osteosarcoma cell line 1 (HOSS1; osteosarcoma) that was primary cultured from a patient-derived xenograft (PDX). Anticancer effects of various chemotherapeutic agents were studied and $\mathrm{IC}_{50}$ values were compared between conventional $2 \mathrm{D}$ and $3 \mathrm{D}$ cell cultures. We provide evidence of cell adhesion mediating chemo-resistance, a new hypothesis relating chemo-resistance to the microenvironment such as the stroma and ECM, which was proposed by Hazlehurst et al. [17]. In addition, we found that sarcoma cells in the 3D model were more radio-resistant, and observed more stromal rich or adhesion-rich phenotypes. A better understanding of how these tumors originate and progress is needed for the development of targeted sarcoma therapies.

\section{Methods}

\section{Cell culture}

The following well-characterized human sarcoma cell lines (American Type Culture Collection) were used in this study: RD, HT1080, and SW872. We also used HOSS1 cells as described in Additional file 1: Supplemental Method. The cells were cultured under standard culture conditions $\left(5 \% \mathrm{CO}_{2}\right.$ at $\left.37^{\circ} \mathrm{C}\right)$ in Dulbecco's modified Eagle's medium (DMEM)/F12 or phenol red-free DMEM/F12 (Gibco, USA) containing $10 \%$ fetal bovine serum (FBS, Invitrogen, USA).

\section{D culture}

Cells were treated with trypsin and counted. Subsequently, the cells were seeded into round-bottom Ultra-Low Attachment 96 well-plates (Corning, Acton, USA) at 200 cells/well in $100 \mu \mathrm{l}$ DMEM-F12 or phenol red-free DMEM-F12 containing $20 \mathrm{ng} / \mathrm{ml}$ epidermal growth factor (EGF), $20 \mathrm{ng} / \mathrm{ml}$ basic fibroblast growth factor (bFGF), $10 \mathrm{ng} / \mathrm{ml}$ hepatocyte growth factor (HGF), $10 \%$ B27, $2 \%$ bovine pituitary extract (BPE), and $20 \%$ methylcellulose as described in Supplemental Method. Spheroids were grown under standard culture conditions (5\% CO at $37^{\circ} \mathrm{C}$ ) and harvested at various time points for RNA isolation or drug testing as described below.

\section{Cell proliferation assay}

3D culture spheres or 2D culture clones were collected and trypsinized with $0.02 \mathrm{M}$ EDTA to single cell. Cells were counted with Countess (Life Technologies, USA) each two days. Cell generation time $(\mathrm{GT}=\mathrm{Tn} / 5 ; \mathrm{Tn}=\mathrm{t} /$ $\ln (\mathrm{Nf} / \mathrm{Ni}) / \ln 2$; Nf: final cell number at time t; $\mathrm{Ni}$ : initial plated cell number; ln: natural logarithm, 5 replicates) was calculated by plotting the graph of cell counting versus time. This experiment was repeated in triplicate.

\section{CDNA microarray}

Equal amounts of cDNA from HOSS1 cultured in 2D and 3D cells were labeled with Cy3, respectively, and were then carried out hybridization according to the manufacture's protocol (Affymetrix) using Affymetriix human genome gene chip HG133 Plus 2.0 (Oebiotech Co, Ltd., Shanghai, China). Genes were considered to be up or down-regulated when the fluorescent intensity 
ratio between $2 \mathrm{D}$ and $3 \mathrm{D}$ cells was greater than 2 or less than 0.5. The experiment was repeated once. Hierarchical clustering of regulated genes in this study was measured by Genespring software.

\section{RNA isolation and RT-qPCR analysis}

Cells or spheroids were collected, washed once with cold PBS, and processed for total RNA isolation using an RNeasy Mini Kit (Qiagen, Germany). RNA integrity and concentrations were analyzed by agarose gel electrophoresis and a Nano-drop Spectrophotometer. One microgram of total RNA was reverse transcribed by M-MLV (Invitrogen, USA) (see Supplemental Method). SYBR-Green Technology (Toyobo, Japan) was used for all RT-qPCR experiments. Further detailed information regarding RT-qPCR is provided in Supplemental Method. Primers were designed by the PCR primer database of Gene Runner and purchased from Invitrogen, which is described in Additional file 2: Table S1.

\section{SDS-polyacrylamide gel electrophoresis (PAGE) and western blotting}

Cell lysates of 2D and 3D cultures were prepared using RIPA lysis buffer. The protein concentrations were measured using a BCA Protein Assay kit (Pierce, USA). Proteins samples $(50 \mu \mathrm{g})$ were resolved by $10 \%$ SDS-PAGE and electrophoretically transferred to PVDF membranes. The membranes were blocked with $5 \%$ fat-free dry milk in TPBS and then incubated for $1 \mathrm{~h}$ at room temperature with primary antibodies (see Additional file 2: Table S2). The primary antibodies were detected with peroxidaseconjugated goat anti-rabbit or anti-mouse IgGs (Jackson, USA). The signals were detected by enhanced chemiluminescence (Pierce).

\section{Immunofluorescence staining}

Spheroids were harvested at various time points and washed twice with PBS. For immunohistochemistry, spheroids were fixed in $4 \%$ paraformaldehyde, embedded in paraffin, and sectioned at $7 \mu \mathrm{m}$ thicknesses. Prior to blocking in PBS plus Tween and $1 \%$ bovine serum albumin, the sections were boiled in $0.01 \mathrm{M}$ sodium citrate buffer $(\mathrm{pH}$ 6.0) for antigen retrieval. Anti-Ecadherin and anti-N-cadherin antibodies were used as primary antibodies. FITC-labeled anti-rabbit or antimouse secondary antibodies (Jackson) were used to detect primary antibodies using a Leica SP5 laser scanning confocal microscope.

\section{Drug treatments}

For 2D culture, cells were seeded in flat-bottom 48well plates (Costar, USA) at 2500 cells/well in $100 \mu \mathrm{l}$ RPMI-1640 medium containing $10 \%$ FBS. For 3D culture, cells were seeded according to the description for spheroid preparation in complete medium. Doxorubicin (AstraZeneca, UK), Gemcitabine (Lilly, France), and Docetaxel (Sanofi, France) were added to the medium of eight replicates for each time point at the indicated final concentrations.

\section{Radiation resistance assay}

For 2D culture, a colony formation assay was performed to determine the sensitivity of cells to X-ray irradiation. Two hundred cells were seeded in each $35-\mathrm{mm}$ dish and allowed to attach. The cells were then exposed to $0-8$ Gy $\mathrm{X}$-ray radiation. The control group did not receive any exposure. Colonies were fixed in methanol and stained with Giemsa at day 14 after exposure. A minimum of 50 viable cells were scored as a colony. For 3D culture, cells were seeded in Ultra-Low Attachment 96-well plates according to the above description. Spheroid formation was analyzed at 2 weeks after exposure to $0-8$ Gy X-ray radiation. Cell survival fraction (SF) was based on the formula: $\mathrm{Ne} /(\mathrm{Ce} \times \mathrm{NO} / \mathrm{CO} \times 100 \%)$, Ne: Colony numbers in experimental group; Ce: Plating numbers in experimental group; N0: Colony numbers in control group; C0: Plating numbers in control group. Cell survival curve was obtained by the single-hit multi-target model $(\mathrm{SF}=1-(1-\mathrm{e}-\mathrm{Do} / \mathrm{D}) \mathrm{N})$ using GraphPad Prism 5 version. The parameters of cell survival fraction mean lethal dose value (Do) and extrapolation number $(\mathrm{N})$ were calculated with a 2 Gy irradiation dose (SF2). Higher values indicated a higher radioresistance.

\section{Measurement of apoptosis}

Cells were stained with Annexin V-FITC and Propidium iodide (PI) according to the manufacturer's instructions (Roche, Germany) to determine the percentage of cells undergoing apoptosis. Annexin V and PI staining was assessed by Flow Cytometry (Becton Dickinson, CA, USA). The percentage of apoptotic cells was expressed as the percentage of the total cell population. To confirm the reliability of this assay for 3D-cultured cells, spheroids were disaggregated with TrypLE Express (Invitrogen) without chelators for $15 \mathrm{~min}$ at $37{ }^{\circ} \mathrm{C}$, re-suspended as single cells, and analyzed by Flow Cytometry.

\section{Statistical analysis}

All data were analyzed by SPSS 11.0 statistical software for Windows. The statistical differences in the data were analyzed using a Student's $t$-test with two sided of at least 5 replicates. All experiments were performed at least three times. A $p$-value of less than 0.05 was regarded as statistically significant. 


\section{Results}

Formation of compact 3D spheroids by soft sarcoma cell lines

After successfully cultured HOSS1 cell line (see Additional file 3: Figure S1), followed by supplements for 3D culture [18], we grew the four sarcoma cells in medium containing methylcellulose, a cellulosederived inert compound that enhances spheroid formation, instead of exogenous ECM components (Fig. 1a). We evaluated HT1080, RD, SW872, and HOSS1 cell lines for their ability to form spheroids (Fig. 1b, c). At 8 days after plating, all cell lines were undergoing stable exponential growth with similar doubling times (Fig. 1d). This similarity in doubling times provided comparable experimental conditions for gene and protein expression analyses. In 3D culture, HT1080 cells reached stable proliferation within 4 days, but RD, SW872 and HOSS1 cells required 8 days. All cell lines reached stable proliferation within $48 \mathrm{~h}$ in $2 \mathrm{D}$ culture (Fig. 1e, f). The growth kinetics of spheroid formation was assessed longitudinally. Cells were counted in 2D and 3D cultures at various time points. Compared with conventional $2 \mathrm{D}$ culture, the increase in cell numbers over time indicated no reduction in the proliferation of cells in 3D culture (Fig. 1g).
Identification of genes regulated in 2D and 3D cultures

To gain further insight into the possible mechanisms underlying $3 \mathrm{D}$ culture compared with $2 \mathrm{D}$, we performed cDNA microarray analyses to find global gene expression changes in $3 \mathrm{D}$ system versus $2 \mathrm{D}$. From our analysis, 116 genes were classified as being up-regulated genes with a ratio $\geq 2.0$, and 94 genes were classified as being down-regulated with a ratio $\leq 0.5$ (see Additional file 2: Table S3). Those genes reportedly involved in tumor cell adhesion, gap junction and ECM, such as CHD1, Cx26, Cx43, Cx45, COL1A1, FN1, LOX, ITGB1 and LAMA4, were also up-regulated following $3 \mathrm{D}$ cultures. Interestingly, $\mathrm{ABC}$ transport genes such as $\mathrm{ABCB} 1, \mathrm{ABCC} 1$, $\mathrm{ABCC} 3, \mathrm{ABCG} 2$, functional chemo-resistance by pumping out drug molecules, were also enhanced in 3D cultures. We were next interested in expression of cadherin, gap junctions, ECM in $3 \mathrm{D}$ system according to sensitive $A B C$ transport and anti-apoptosis genes reflected chemo-resistance in 3D platform.

\section{Expression of adhesion and gap junction molecules in 3D cultures}

To assess cellular interactions, we detected the expression of adhesion and gap junction molecules in cells after 8 days of spheroid formation. First, gap junctions were identified between adjacent cells. An abundance of

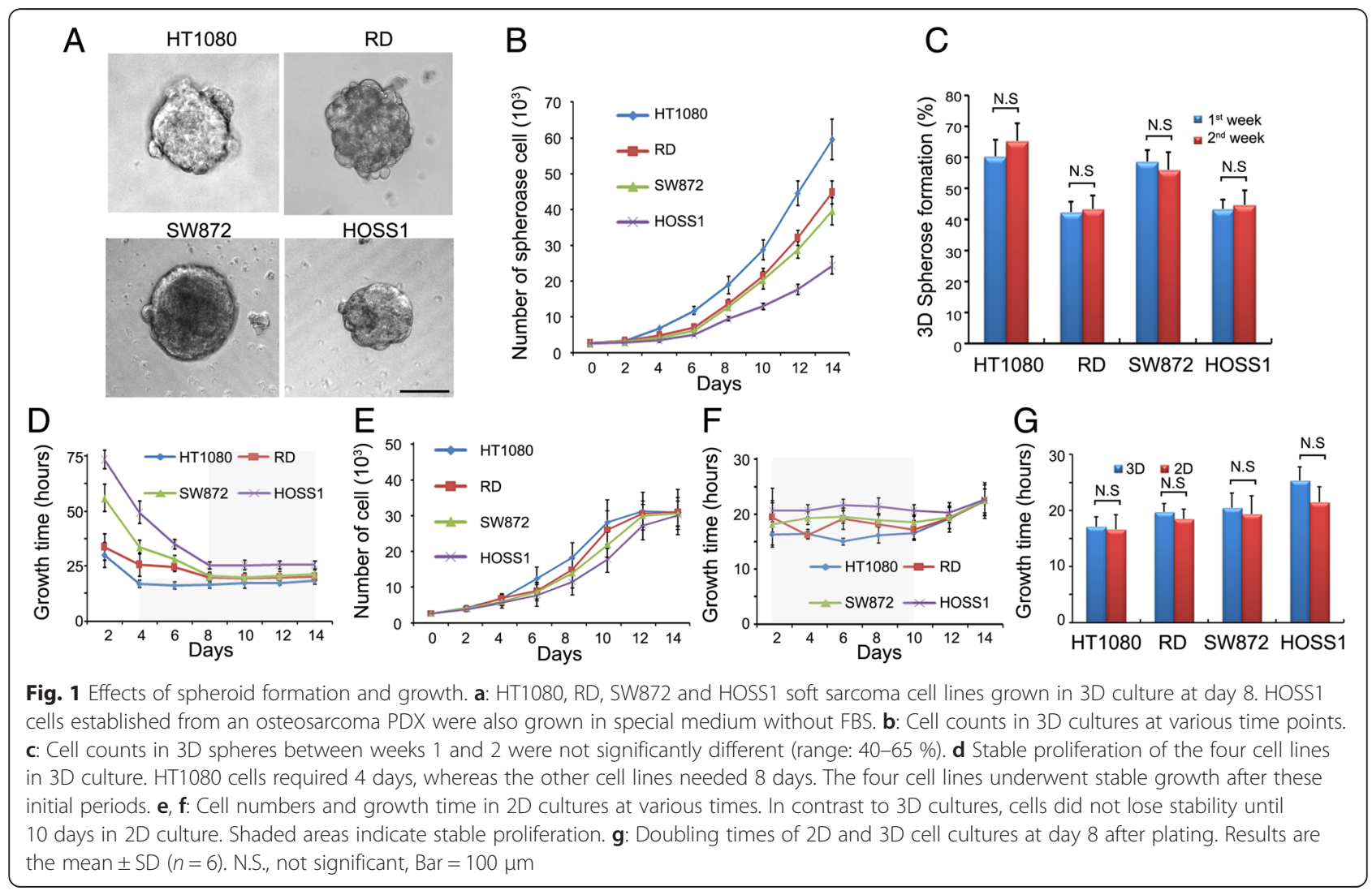


Cx26, Cx43, and Cx45 was detected in HOSS1 cell spheroids, which were absent in 2D cultures (Fig. 2a). These results were supported by RT-qPCR and western blot analyses of HOSS1 cells (Fig. 2b, c). Interestingly, compared with 2D cultures, mRNA expression of $\mathrm{N}$ cadherin in HOSS1 cells increased in 3D cultures during the initial phase of spheroid formation and then decreased after 8 days (Fig. $2 \mathrm{~d}$, e). In contrast, both the mRNA and protein expression of E-cadherin was weak in the earlier period of spheroid formation, and then increased at later stages when the cells had proliferated as spheroids after day 6 (Fig. 2f). In addition, because of the cell-cell contacts over the entire cell surface, Ecadherin protein expression was consistently higher in $3 \mathrm{D}$ cultures compared with $2 \mathrm{D}$ cultures in which cells only make limited contacts. All target genes were validated in other sarcoma cell, in which Cx26, Cx43, Cx45, E-cadherin and N-cadherin were enhanced both mRNA and protein levels (Additional file 4: Figure S2).

\section{Increases in ECM components of 3D cultures}

Next, we detected the gene and protein expression of stromal ECM components such as collagen and fibronectin-1. mRNA expression of COL1A1, LOX, FN1, SNED1, ITGB1, and LAMA4 was examined by RT-PCR at various time points in $3 \mathrm{D}$ and $2 \mathrm{D}$ cultures (Fig. 3a). The expression of these genes was higher in $3 \mathrm{D}$ cultures during sphere formation and the compaction phase. In confluent 2D cultures, the expression of these genes did not increase. Expression of collagen I and fibronectin-1, which mediate cell and tissue cohesion, was confirmed by western blot analysis of spheroids. Lysyl oxidase (LOX), a proteoglycan with high expression in many cancerous tissues, is found in the intercellular and extracellular stroma of soft sarcomas. We detected high expression of LOX in 3D cultures, whereas LOX was only expressed in 2D cultures when the cells became confluent. Expression of SNED1 (sushi-nidogen and EGF-like domains 1) was strongly up-regulated in 3D culture (Fig. 3b). Interestingly, some studies have considered SNED1 as a cisplatin resistance-related gene in head and neck squamous carcinoma [19]. Therefore, it may possibly reflect chemo-resistance in 3D spheroids.

\section{Chemo- and radio-resistances in 3D cultures}

We detected the mRNA and protein expression of $\mathrm{ABCB} 1, \mathrm{ABCC} 1$, and $\mathrm{ABCG} 2$ in $3 \mathrm{D}$ cultures for comparison with $2 \mathrm{D}$ cultures. Most of these ABC transporters were up-regulated during spheroid formation (Fig. 4a, b). Next, we examined the differences in the sensitivities to Doxorubicin [20], Gemcitabine [21] and Docetaxel [22] applied to 3D and 2D cultures. Each clone formation of the three drugs was detected in both $2 \mathrm{D}$ and $3 \mathrm{D}$ cultures of HOSS1 cells (Fig. 4c). And $\mathrm{IC}_{50}$ of three drugs were calculated in $2 \mathrm{D}$ and $3 \mathrm{D}$ culture with all four soft sarcoma cell lines (see Table 1). The

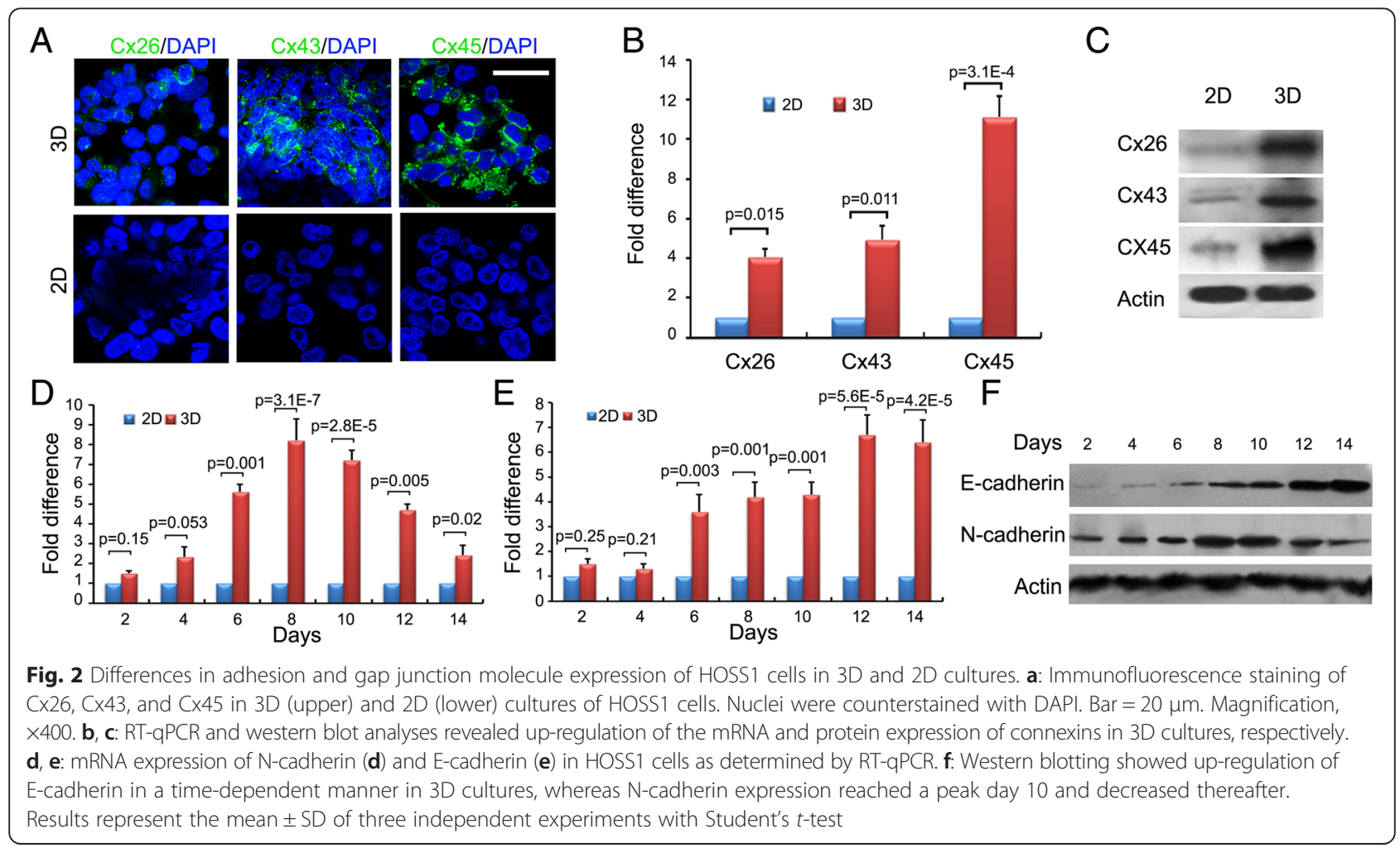



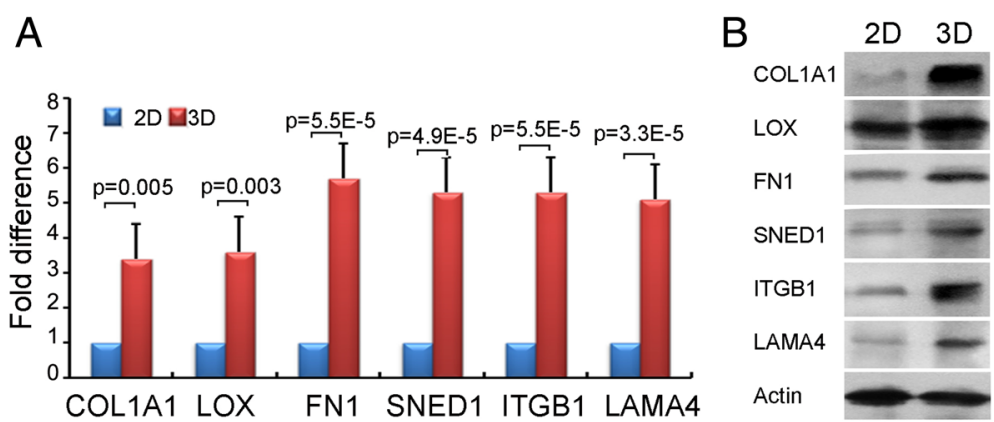

Fig. 3 ECM-related gene expressions of HOSS1 cells in 3D and 2D cultures. a: RT-qPCR analysis of the expression of ECM-related genes in 3D and 2D cultures of HOSS1 cells at day 8. Data are presented as fold differences relative to 2D-cultured cells for each gene, which was defined as 1 (calibrator). Data are mean \pm SD of 3 independent experiments with Student's t-test. Error bars indicate SD. b: Western blotting of ECM-related protein expression in 3D and 2D cultures at day 8

reasons are related to the specific interactions between cells and their microenvironments, namely cell-cell and cell-ECM interactions, which maintain the effects of chemotherapy. Additionally, we clarified the effect on radio-resistance under $3 \mathrm{D}$ conditions. Cells in 3D culture had significantly higher clonogenic survival upon exposure to increasing single doses of X-ray radiation from 1 to 8 Gy compared with 2D cultures (Fig. 4d, Table 2).

\section{Apoptosis of 3D spheroids}

In $2 \mathrm{D}$ cultures treated with drugs, we observed a significant decrease in cell proliferation as well as an increase to $50-70 \%$ apoptotic cells in the four sarcoma cell lines.
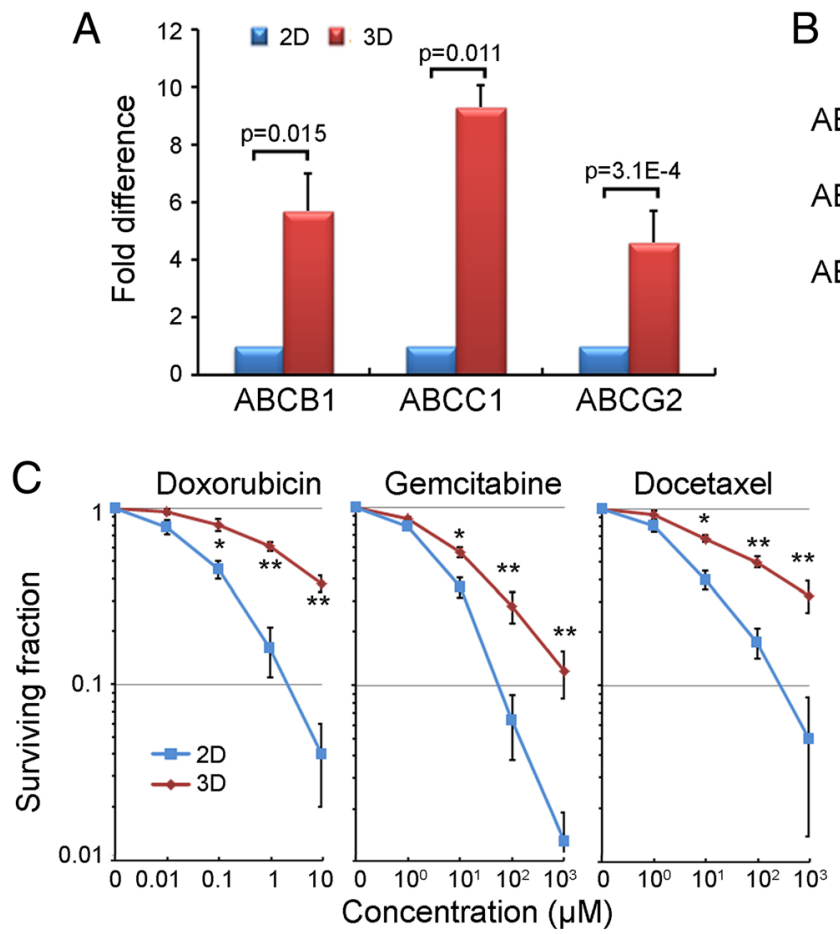

B
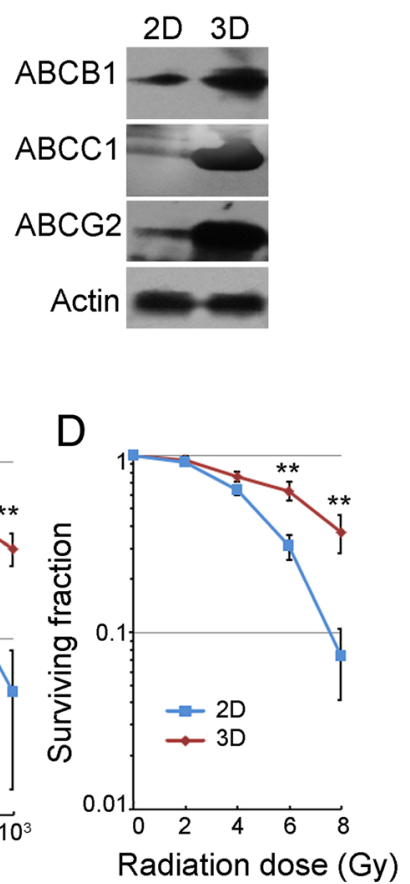

Fig. 4 Increases in chemo-resistances against anticancer agents and radio-resistances in 3D cultures of HOSS1 cells. a: RT-qPCR analysis of drug resistance-related genes (ATP-binding cassette transporters) in 3D and 2D cultures. Values in 2D cultures for each gene were defined as 1. Results are the mean \pm SD $(n=3)$ with Student's-t test. $\mathbf{b}$ : Western blot analysis of HOSS1 cells in 3D and 2D cultures. $\mathbf{c}$, $\mathbf{d}$ : Clonogenic survival of 3D- and 2D-cultured cells exposed to various concentrations of Doxorubicin (10, $100 \mathrm{nM}, 1 \mu \mathrm{M}$, and $10 \mu \mathrm{M})$, Gemcitabine (1, 10, 100, and 1000 $\mu \mathrm{M})$, and Docetaxel $(1,10,100$, and $1000 \mu \mathrm{M})(\mathbf{c})$ or X-ray doses $(2,4,6$, and $8 \mathrm{~Gy})(\mathbf{d})$ at 24 hours after plating. ${ }^{*} P<0.05{ }^{* *} P<0.01$ vs. respective 2Dcultured cells. Results of a representative independent experiment of three are shown 
Table 1 Comparative analysis of $\mathrm{IC}_{50}(\mu \mathrm{M})$ values of various anticancer drugs in $2 \mathrm{D}$ and $3 \mathrm{D}$ cultures

\begin{tabular}{|c|c|c|c|c|c|c|}
\hline \multirow[t]{2}{*}{ Cells } & \multicolumn{2}{|l|}{ Doxorubicin } & \multicolumn{2}{|c|}{ Gemcitabine } & \multicolumn{2}{|l|}{ Docetaxel } \\
\hline & $2 \mathrm{D}$ & $3 \mathrm{D}$ & $2 \mathrm{D}$ & $3 \mathrm{D}$ & $2 \mathrm{D}$ & $3 \mathrm{D}$ \\
\hline HT1080 & $0.025 \pm 0.002$ & $4.27 \pm 0.38^{*}$ & $4.26 \pm 0.58$ & $39.26 \pm 2.76^{*}$ & $2.88 \pm 0.54$ & $69.81 \pm 5.50^{*}$ \\
\hline $\mathrm{RD}$ & $0.014 \pm 0.01$ & $2.74 \pm 0.56^{*}$ & $1.89 \pm 0.64$ & $43.27 \pm 3.32^{*}$ & $1.17 \pm 0.16$ & $55.87 \pm 6.61^{*}$ \\
\hline SW872 & $0.066 \pm 0.04$ & $2.45 \pm 0.72^{*}$ & $2.57 \pm 0.15$ & $18.34 \pm 2.26^{*}$ & $1.85 \pm 0.22$ & $94.48 \pm 10.25^{*}$ \\
\hline HOSS1 & $0.078 \pm 0.011$ & $4.61 \pm 0.33^{*}$ & $6.23 \pm 0.19$ & $23.55 \pm 3.65^{*}$ & $6.72 \pm 0.87$ & $103.2 \pm 7.82^{*}$ \\
\hline
\end{tabular}

Each data point is represented as mean \pm SD $(n=4-6)$

${ }^{*} P<0.01$ Vs repective $2 \mathrm{D}$ cultures

To quantify apoptosis induced by Doxorubicin, Gemcitabine and Docetaxel, we used an Annexin V and PI analysis kit. Remarkably, induction of apoptosis was observed after $24 \mathrm{~h}$ with differences in the sensitivities of HOSS1 cells to the three drugs in 3D and 2D cultures (Fig. 5a). The apoptosis ratio was higher (55-80\%) in $2 \mathrm{D}$ cultures than in $3 \mathrm{D}$ cultures (20-35\%) at the same drug concentrations. Furthermore, similar sensitivities of the four sarcoma cell lines to Doxorubicin, Gemcitabine, and Docetaxel were found in 3D cultures (Fig. 5b, c, d). HT1080, RD, SW872 and HOSS1 cells showed significant decreases in apoptosis by about 20-45\% upon drug treatments at high doses in 3D culture. These data showed a limited response of apoptosis upon highconcentration drug treatments in most soft sarcoma cells in 3D culture.

\section{Discussion}

Because most experiments have used cells grown in 2D monolayer cultures, in this study we analyzed the effect of cell-cell communication and more physiological settings on soft sarcoma formation by employing a 3D culture system. In 3D platform, sarcoma cell growth more closely mimicked the in vivo pathological process [23]. The cells appeared to be healthy when cultured in the 3D environment in vitro under our cultured conditions. We employed culture supplements and methylcellulose, but not matrigel or agarose, because these factors did not facilitate trypsinization of spheroids to count the number of cells accurately. Certain growth factors used in stem cell culture medium, such as EGF and bFGF,

Table 2 Radio-sensitivity parameters of different cell lines in 2D and 3D cultures

\begin{tabular}{lllllll}
\hline Cells & SF2 & \multicolumn{3}{c}{ D } \\
& 2D & 3D & $2 \mathrm{D}$ & $3 \mathrm{D}$ & $2 \mathrm{D}$ & $3 \mathrm{D}$ \\
\hline HT1080 & 0.78 & 0.95 & 1.51 & 2.27 & 2.7 & 4.8 \\
RD & 0.81 & 0.91 & 2.34 & 2.74 & 2.1 & 4.6 \\
SW872 & 0.77 & 0.84 & 1.86 & 3.45 & 2.8 & 5.1 \\
HOSS1 & 0.89 & 0.94 & 2.28 & 3.8 & 2.6 & 4.8 \\
\hline
\end{tabular}

SF2: cell survival fraction in 2Gy irradiation dose; Do: mean lethal dose value; $\mathrm{N}$ : extrapolation number were used to prevent cell differentiation and maintain cell growth [24]. In this system, cell spheres formed well, but the time needed for cell growth stableness (in logarithmic growth phase) was about 8 days in 3D culture, whereas only 2 days were required in $2 \mathrm{D}$ culture, because the single cells needed more time to adapt to the 3D environment. Upon re-establishment of cell-cell communication in spheres, the cell signals are distributed appropriately, and the cells grow in more similar manner as their original source.

Using gene chip analysis, we identified 116 genes that were up-regulated and 94 genes that were downregulated in $3 \mathrm{D}$ culture compared with $2 \mathrm{D}$ cultured cells. These genes belong to the various categories of signal transduction, transcription factor, cell cycle, cell growth, motility, apoptosis and differentiation, as well as gap junction, cell adhesion and ECM compositions.

Beginning, in our 3D model, we found expression of connexins Cx26, Cx43 and Cx45, which affected the proliferation of sarcoma cells, whereas their expression was lost in 2D culture. Many studies have shown that gap junction molecules such as Cx43 contribute to cellcell interactions, although their expression differs in various cell types [25-27]. Currently, it is unclear why connexins were re-expressed in $3 \mathrm{D}$ culture. However, immunofluorescences staining showed expression of gap junction proteins in 3D culture were raised up, indicating a change in the cellular phenotype. It suggests that the expression of connexins depends on the environment and the effects of biological factors.

Besides, the expression of cadherin, cell-cell adhesion molecules, has been reported in some sarcomas. Both Ecadherin and $\mathrm{N}$-cadherin are weakly expressed in sarcoma in $2 \mathrm{D}$ cultures as reported previously [28-30]. However, in our 3D cultures, both of these molecules were upregulated and increased the cell-cell communication of sarcoma cells. There were also cell line-specific differences that further highlight the importance of testing the effects of various factors in a proper $3 \mathrm{D}$ environment.

Last, when cells form spheres, the composition of the ECM, such as collagen, laminin, and fibronectin, contribute to the remolding of ECM. For example, it has been reported that laminin A4 is associated with 


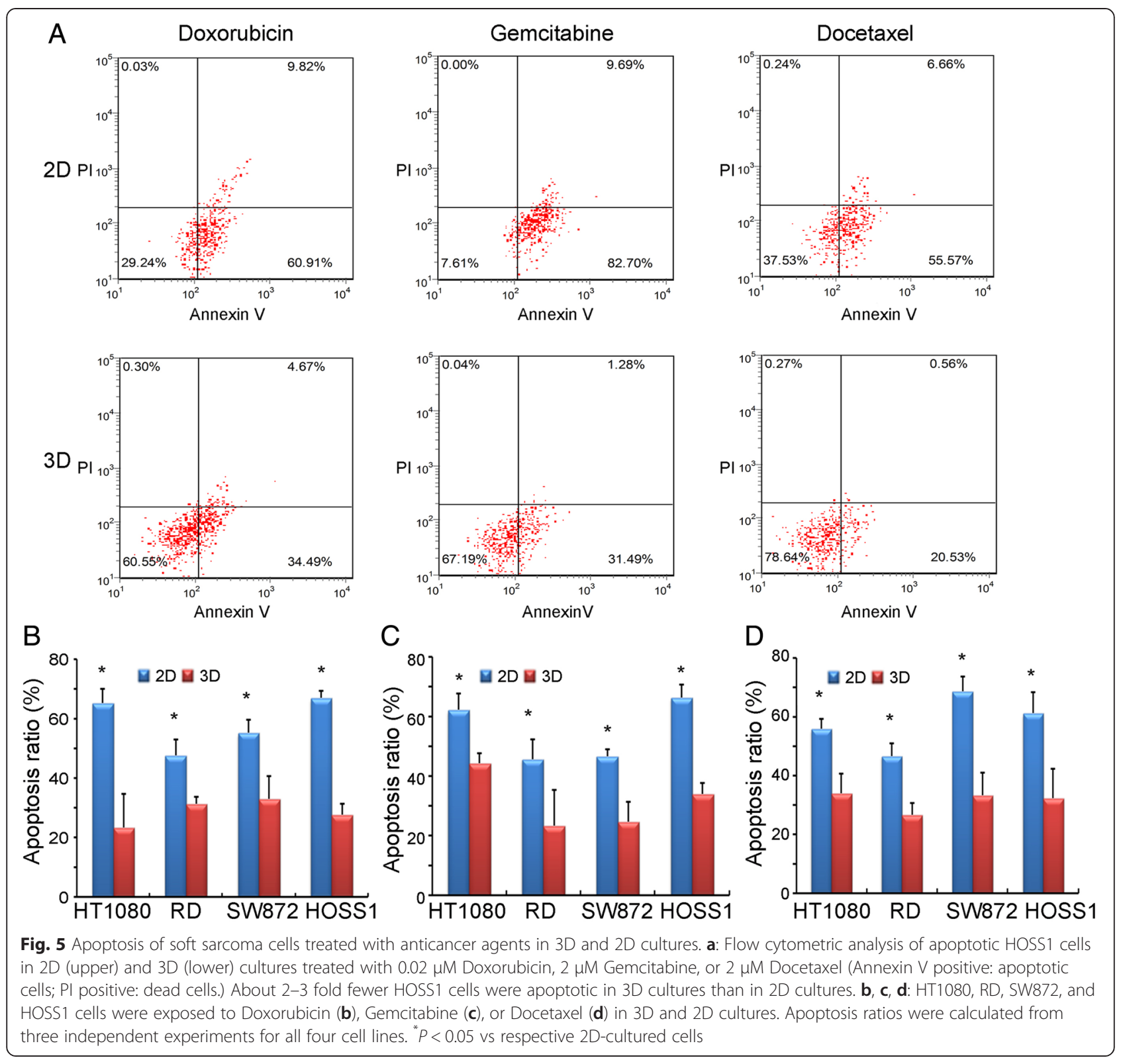

metastasis in some soft sarcoma cells according to influence tumor microenvironments [31]. Biologically active molecules related to the composition of the microenvironment, such as LOX, have been shown to be up-regulated in soft sarcoma cells [32, 33]. It would be functionally cross-linking collagen to contribute increasing the stiffness in $3 \mathrm{D}$ culture. It is the reason that the remolding of ECM is so necessary to $3 \mathrm{D}$ cultures of sarcoma cells.

On functional level, the growth response of cells to chemotherapeutic drugs and radiation treatment in $3 \mathrm{D}$ culture was similar to that of cells in 2D culture. However, we observed more chemo and radio resistance in medium containing methylcellulose of 3D culture. These data could be explained by several factors. First, the restoration of defective gap junctions in cancer could promote re-sensitization to current chemotherapeutics [34]. In human spheroidal cultures, gap junctions were identified as mediators of tumorigenicity by influencing E-cadherin expression [35]. Second, loss of E-cadherin involved in the regulation of EMT and metastasis, and its roles in both drug and immune resistance point out that Snail is a target for sensitization to cytotoxic drugs in cadherin signal pathway [36]. Third, remolding of ECM is benefited for protecting cells from chemotherapy and radiotherapy [37-39]. These factors contribute to chemo- and radio-resistance depended on the microenvironments in 3D culture.

Furthermore, in addition to factors in the stromal microenvironment, $\mathrm{ABC}$ transporters play an important role in chemo-resistance by pumping out drug molecules 
[40]. Three major $\mathrm{ABC}$ transporters, $\mathrm{ABCB} 1, \mathrm{ABCC} 1$, and ABCG2, were upregulated at both gene and protein levels in $3 \mathrm{D}$ cultures, which contributes to chemo-resistance. In our study, the cellular response to drugs was affected by microenvironmental factors, and 3D culture appeared to be more appropriate to detect chemo-resistances. Our results confirmed increases in the chemo-resistance of the four sarcoma cell lines. The reason why higher concentrations of drugs, which are used to treat sarcoma in clinical therapy, were present in the $3 \mathrm{D}$ model is possibly explained by more abundant $\mathrm{ABC}$ transporters and the enhanced biologically active molecules in 3D culture. Acquiring new phenotypes as a result of alterations in the microenvironmental composition is a major factor protecting cells from drug activities. Moreover, we examined radio-resistance against X-rays. Most soft sarcomas are not sensitive to X-ray irradiation, but their resistance increases in growing spheroids [41]. Our data suggest a connection between growth conditions and cellular chemo- and radio-resistances.

Accumulation of Doxorubicin, Gemcitabine, and Docetaxel caused apoptosis as detected by Annexin V and PI staining. The three drugs could not sensitize cells to undergo the early period of apoptosis in $3 \mathrm{D}$ culture, but contributed to a significant increase in apoptosis of cells in 2D cultures. The dysfunction of apoptosis may result from growth in the 3D environment, which is probably the reason for the radio-resistance.

In summary, 3D culture models are being increasingly employed in cancer research, as well as in tissue engineering and developmental and cell biology. To develop therapies, cell responsiveness is an important issue, and there are major differences in 3D physiological microenvironments and $2 \mathrm{D}$ culture. Why does the positive anticancer effect observed in 2D cultures often fail during in vivo testing? One of the reasons is that we ignored cell-cell communications in tumor spheres formation. As the results, the ability to predict outcomes in preclinical animal experiments and clinical trials can be better understood using 3D cultures, which is a potentially useful predictive tool in clinic anti-sarcoma screening in the future. Using 3D spheroid cultures from clinic surgical patients can be expanded to various fields of drug sensitive test, radio sensitive test, novel drug delivery approaches and X-ray project design for personal antisarcoma therapeutic strategy.

\section{Conclusion}

Presently, a limitation of the 3D model is the number of cell populations and supplements. Accordingly, this model requires more refined tools to examine the adhesion, gap junction, ECM and apoptosis associated with drug- and radio-resistances in soft sarcoma.

\section{Additional files}

\section{Additional file 1: Supplemental Methods. \\ Additional file 2: Supplemental Tables. PCR primers, Antibodies and Gene Chip Data.}

Additional file 3: Figure S1. Primary culture HOSS1 cell line from soft-sarcoma PDX. (A): Representative photograph shown soft-sarcoma formation in NOD/SCID mice from patient derived xenograft.Arrow shown sarcoma formats by subcutaneous injection. (B): The histology of PDX tissues was verified from original patient by H\&E staining. (C): morphology of HOSS1 cell line was taken by phase contrast microscope. Bar $=50 \mu \mathrm{m}$, Magnification, $\times 400$.

Additional file 4: Figure S2. mRNA and protein levels in sarcoma cells. (A), (B) and (C): mRNA levels of target genes were shown respectively in HT1080, RD and SW872. (D): The proteins were expressed of HT1080, RD and SW872. Results represent the mean \pm SD of three independent experiments with Student's t-test.

\section{Abbreviations}

HOSS1: Human Osteosarcoma cell line-1; PDX: Patient Derived Xenograft; FACS: Fluorescence-activated cell sorting; PI: Propidium iodide; ECM: Extracellular matrix; BPE: Bovine pituitary extract; LOX: Lysyl Oxidase, $I_{50}$, The $50 \%$ inhibitory concentration.

\section{Competing interests}

The authors declare that they have no competing interests.

\section{Authors' contributions}

For research articles with several authors, CB and ZF conceived and designed the experiments; CB and MY performed the experiments; MY established PDX model and primary culture HOSS1; ZF analyzed the data; SL and TG contributed reagents/materials/analysis tools; CB and MY wrote the paper. All authors read and approved the final manuscript.

\section{Acknowledgments}

Thanks are given to the FACS Core Facility of Peking University Cancer Hospital for performing FACS assays. This study was supported by the funder of Peking University Cancer Hospital (No. 08-29).

\section{Author details}

${ }^{1}$ Department Bone and Soft Tissue Tumor, Key Laboratory of Carcinogenesis and Translational Research (Ministry of Education), Peking University Cancer Hospital and Institute, Beijing 100142, People's Republic of China.

${ }^{2}$ Department of Gerontology, Beijing Shijitan Hospital, Capital Medical

University, Beijing 100038, People's Republic of China.

Received: 17 March 2015 Accepted: 21 May 2015

Published online: 10 June 2015

\section{References}

1. Egas-Bejar D, Huh WW. Rhabdomyosarcoma in adolescent and young adult patients: current perspectives. Adolesc Med Health Therapeutics. 2014;5:115-25.

2. Coffin CM, Alaggio R. Fibroblastic and myofibroblastic tumors in children and adolescents. Pediatr Dev Pathol. 2012;15(1 Suppl):127-80.

3. Vijayamurugan N, Bakhshi S. Review of management issues in relapsed osteosarcoma. Expert Rev Anticancer Ther. 2014;14(2):151-61.

4. Wang D, Abrams RA. Radiotherapy for soft tissue sarcoma: 50 years of change and improvement. Am S Clinical Oncology Educational book / ASCO Am Soc Clinical Oncology Meeting 2014:244-51. doi:10.14694/ EdBook_AM.2014.34.244.

5. Patel SR. Fifty years of advances in sarcoma treatment: moving the needle from conventional chemotherapy to targeted therapy. Am Soc Clinical Oncology educational book / ASCO Am Society of Clinical Oncology Meeting. 2014:259-62. doi:10.14694/EdBook_AM.2014.34.259.

6. Lohi O, Jahnukainen K, Huttunen P, Taskinen M, Taskinen S, Pakarinen M, et al. Solid tumors in children. Duodecim. 2014;130(20):2050-9. 
7. Hirt C, Papadimitropoulos A, Mele V, Muraro MG, Mengus C, lezzi G, et al. "In vitro" 3D models of tumor-immune system interaction. Adv Drug Deliv Rev. 2014;79-80:145-54.

8. Levinger I, Ventura Y, Vago R. Life is three dimensional-as in vitro cancer cultures should be. Adv Cancer Res. 2014;121:383-414.

9. Sutherland RM, Durand RE. Radiation response of multicell spheroids-an in vitro tumour model. Curr Top Radiat Res Q. 1976;11(1):87-139.

10. Fong EL, Lamhamedi-Cherradi SE, Burdett E, Ramamoorthy V, Lazar AJ, Kasper FK, et al. Modeling Ewing sarcoma tumors in vitro with 3D scaffolds. Proc Natl Acad Sci U S A. 2013;110(16):6500-5.

11. Mesri EA, Cesarman E. Kaposi's sarcoma herpesvirus oncogenesis is a notch better in 3D. Cell Host Microbe. 2011;10(6):529-31.

12. Fourre N, Millot JM, Garnotel R, Jeannesson P. In situ analysis of doxorubicin uptake and cytotoxicity in a 3D culture model of human HT-1080 fibrosarcoma cells. Anticancer Res. 2006;26(6B):4623-6.

13. Hehlgans S, Eke I, Storch K, Haase M, Baretton GB, Cordes N. Caveolin-1 mediated radioresistance of 3D grown pancreatic cancer cells. Radiother Oncol. 2009;92(3):362-70.

14. Jiguet Jiglaire C, Baeza-Kallee N, Denicolai E, Barets D, Metellus P, Padovani $L$, et al. Ex vivo cultures of glioblastoma in three-dimensional hydrogel maintain the original tumor growth behavior and are suitable for preclinical drug and radiation sensitivity screening. Exp Cell Res. 2014;321(2):99-108

15. Niero EL, Rocha-Sales B, Lauand C, Cortez BA, de Souza MM, RezendeTeixeira $P$, et al. The multiple facets of drug resistance: one history, different approaches. J Exp Clin Cancer Res. 2014;33:37.

16. Kretschmer N, Rinner B, Stuendl N, Kaltenegger H, Wolf E, Kunert O, et al. Effect of costunolide and dehydrocostus lactone on cell cycle, apoptosis, and $\mathrm{ABC}$ transporter expression in human soft tissue sarcoma cells. Planta Med. 2012;78(16):1749-56.

17. Hazlehurst LA, Landowski TH, Dalton WS. Role of the tumor microenvironment in mediating de novo resistance to drugs and physiological mediators of cell death. Oncogene. 2003;22(47):7396-402.

18. Chitcholtan K, Asselin E, Parent S, Sykes PH, Evans JJ. Differences in growth properties of endometrial cancer in three dimensional (3D) culture and 2D cell monolayer. Exp Cell Res. 2013;319(1):75-87.

19. Leimeister C, Schumacher N, Diez H, Gessler M. Cloning and expression analysis of the mouse stroma marker Snep encoding a novel nidogen domain protein. Dev Dyn. 2004;230(2):371-7.

20. Sugiyama I, Sadzuka Y. Enhanced antitumor activity of different double arms polyethyleneglycol-modified liposomal doxorubicin. Int J Pharm. 2013;441(1-2):279-84.

21. Schmitt T, Kosely F, Wuchter P, Schmier JW, Ho AD, Egerer G. Gemcitabine and docetaxel for metastatic soft tissue sarcoma - a single center experience. Onkologie. 2013;36(7-8):415-20

22. Kaya AO, Buyukberber S, Ozkan M, Alkis N, Sevinc A, Ozdemir NY, et al. Efficacy and toxicity of gemcitabine plus docetaxel combination as a second line therapy for patients with advanced stage soft tissue sarcoma. Asian Pac J Cancer Prev. 2012;13(2):463-7.

23. Longati $P$, Jia X, Eimer J, Wagman A, Witt MR, Rehnmark S, et al. 3D pancreatic carcinoma spheroids induce a matrix-rich, chemoresistant phenotype offering a better model for drug testing. BMC Cancer. 2013;13:95.

24. Nieto-Estevez V, Pignatelli J, Arauzo-Bravo MJ, Hurtado-Chong A, VicarioAbejon C. A global transcriptome analysis reveals molecular hallmarks of neural stem cell death, survival, and differentiation in response to partial FGF-2 and EGF deprivation. PLoS One. 2013;8(1). e53594.

25. Talbot J, Brion R, Picarda G, Amiaud J, Chesneau J, Bougras G, et al. Loss of connexin43 expression in Ewing's sarcoma cells favors the development of the primary tumor and the associated bone osteolysis. Biochim Biophys Acta. 2013;1832(4):553-64.

26. Yoshitani K, Kido A, Honoki K, Akahane M, Fujii H, Tanaka Y. Low concentrations of alendronate increase the local invasive potential of osteoblastic sarcoma cell lines via connexin 43 activation. Pathol Res Pract. 2011;207(7):417-22.

27. King TJ, Fukushima LH, Yasui Y, Lampe PD, Bertram JS. Inducible expression of the gap junction protein connexin43 decreases the neoplastic potential of HT-1080 human fibrosarcoma cells in vitro and in vivo. Mol Carcinog. 2002;35(1):29-41

28. Chaklader M, Pan A, Law A, Chattopadhayay S, Chatterjee R, Law S. Differential remodeling of cadherins and intermediate cytoskeletal filaments influence microenvironment of solid and ascitic sarcoma. Mol Cell Biochem. 2013;382(1-2):293-306
29. Sun C, Wang Z, Zheng Q, Zhang H. Salidroside inhibits migration and invasion of human fibrosarcoma HT1080 cells. Phytomedicine. 2012;19(3-4):355-63.

30. Guo HB, Johnson H, Randolph M, Pierce M. Regulation of homotypic cell-cell adhesion by branched N-glycosylation of N-cadherin extracellular EC2 and EC3 domains. J Biol Chem. 2009;284(50):34986-97.

31. Gorelik JV, Cherepanova OA, Voronkina IV, Diakonov IA, Blinova MI, Pinaev GP. Laminin-2/4 from human placenta is a better adhesion agent for primary keratinocytes than laminin-1 from EHS sarcoma. Cell Biol Int. 2001;25(5):395-402.

32. Liu Y, Lv B, He Z, Zhou Y, Han C, Shi G, et al. Lysyl oxidase polymorphisms and susceptibility to osteosarcoma. PLoS One. 2012;7(7):e41610.

33. $X u X$, Wang $B, X u Y$. Expression of lysyl oxidase in human osteosarcoma and its clinical significance: a tumor suppressive role of LOX in human osteosarcoma cells. Int J Oncol. 2013;43(5):1578-86.

34. Forster $T$, Rausch V, Zhang Y, Isayev O, Heilmann K, Schoensiegel F, et al. Sulforaphane counteracts aggressiveness of pancreatic cancer driven by dysregulated Cx43-mediated gap junctional intercellular communication Oncotarget. 2014;5(6):1621-34.

35. Yu SC, Xiao HL, Jiang XF, Wang QL, Li Y, Yang XJ, et al. Connexin 43 reverses malignant phenotypes of glioma stem cells by modulating E-cadherin. Stem Cells. 2012;30(2):108-20.

36. Kaufhold S, Bonavida B. Central role of Snail1 in the regulation of EMT and resistance in cancer: a target for therapeutic intervention. J Exp Clin Cancer Res. 2014;33:62.

37. Legant WR, Chen CS, Vogel V. Force-induced fibronectin assembly and matrix remodeling in a 3D microtissue model of tissue morphogenesis. Integr Biol. 2012;4(10):1164-74.

38. Katikaneni R, Ponnapakkam T, Suda H, Miyata S, Sakon J, Matsushita O, et al. Treatment for chemotherapy-induced alopecia in mice using parathyroid hormone agonists and antagonists linked to a collagen binding domain. Int J Cancer J Int Du Cancer. 2012;131(5):E813-21.

39. Storch K, Cordes N. Focal adhesion-chromatin linkage controls tumor cell resistance to radio- and chemotherapy. Chemother Res Pract. 2012;2012:319287.

40. Yu M, Ocana A, Tannock IF. Reversal of ATP-binding cassette drug transporter activity to modulate chemoresistance: why has it failed to provide clinical benefit? Cancer Metastasis Rev. 2013:32(1-2):211-27.

41. Yuan M, Eberhart CG, Kai M. RNA binding protein RBM14 promotes radio-resistance in glioblastoma by regulating DNA repair and cell differentiation. Oncotarget. 2014;5(9):2820-6

\section{Submit your next manuscript to BioMed Central and take full advantage of:}

- Convenient online submission

- Thorough peer review

- No space constraints or color figure charges

- Immediate publication on acceptance

- Inclusion in PubMed, CAS, Scopus and Google Scholar

- Research which is freely available for redistribution

Submit your manuscript at www.biomedcentral.com/submit

C BioMed Central 